\title{
THE HERCULES CLUSTER OF GALAXIES
}

M. TARENGHI

Laboratorio di Fisica Cosmica e Tecnologie Relative C.N.R. Milano - Italy

De nouvelles observations de décalage spectral et de la morphologie d'un échantillon de galaxies plus brillantes que $\mathrm{m}_{\mathrm{p}}=15.8$ sont pratiquement achevées dans un cercle de $2.5^{\circ}$ degrayon autour du centre du complexe de galaxies d'Hercule. Les résultats préliminaires suggèrent l'existence d'un superamas d'Hercule.

This communication presents preliminary results of a morphological and spectroscopic study of galaxies in a large area including the Hercules cluster of galaxies done by W.G. Tifft, G. Chincarini, H.J. Rood, L. Thompson and myself. For the morphological study we have used plates obtained at the prime focus of the 4-meter telescope of Kitt Peak National Observatory, plates obtained with a 140mm ITT image tube attached to the 90" Steward Telescope, and moreover we have used two IIIa-J 48" Palomar Schmidt plates borrowed from $A$. Oemler. The spectroscopic material has been obtained with the $90 "$ "Steward telescope, the 84" Kitt Peak telescope and the 107" McDonald telescope, over a period of time of three years. Generally a Cassegrain spectrograph with an image tube and a $300 \mathrm{line} / \mathrm{mm}$ grating was used giving dispersions of about $240 \AA / \mathrm{mm}$ in the blue.

Figure I shows the region studied. It covers a large fraction of the cluster number 7 in the field 108 of the Zwicky and Herzog (1963) catalogue. This cluster centered at $\alpha(1950)=$ $16^{\mathrm{h}} 00^{\mathrm{m}} 24^{\mathrm{s}}, \delta(1950)=19^{\circ} 25$ is described as a nearby medium compact cluster with a population of 2859 galaxies and an equivalent angular diameter of $6^{\circ} .4$. In the area covered by Zwicky's cluster there are three rich cluster of galaxies listed by Abell (1958) in his catalogue: A2147, A2151 and A2152 Table I gives the coordinates, the distance and richness classes, the magnitude of the 10th brightest galaxy, and the morphological type in the Bautz and Morgan (1970) and Rood and Sastry (1971) classifications of the three rich Abell clusters. 


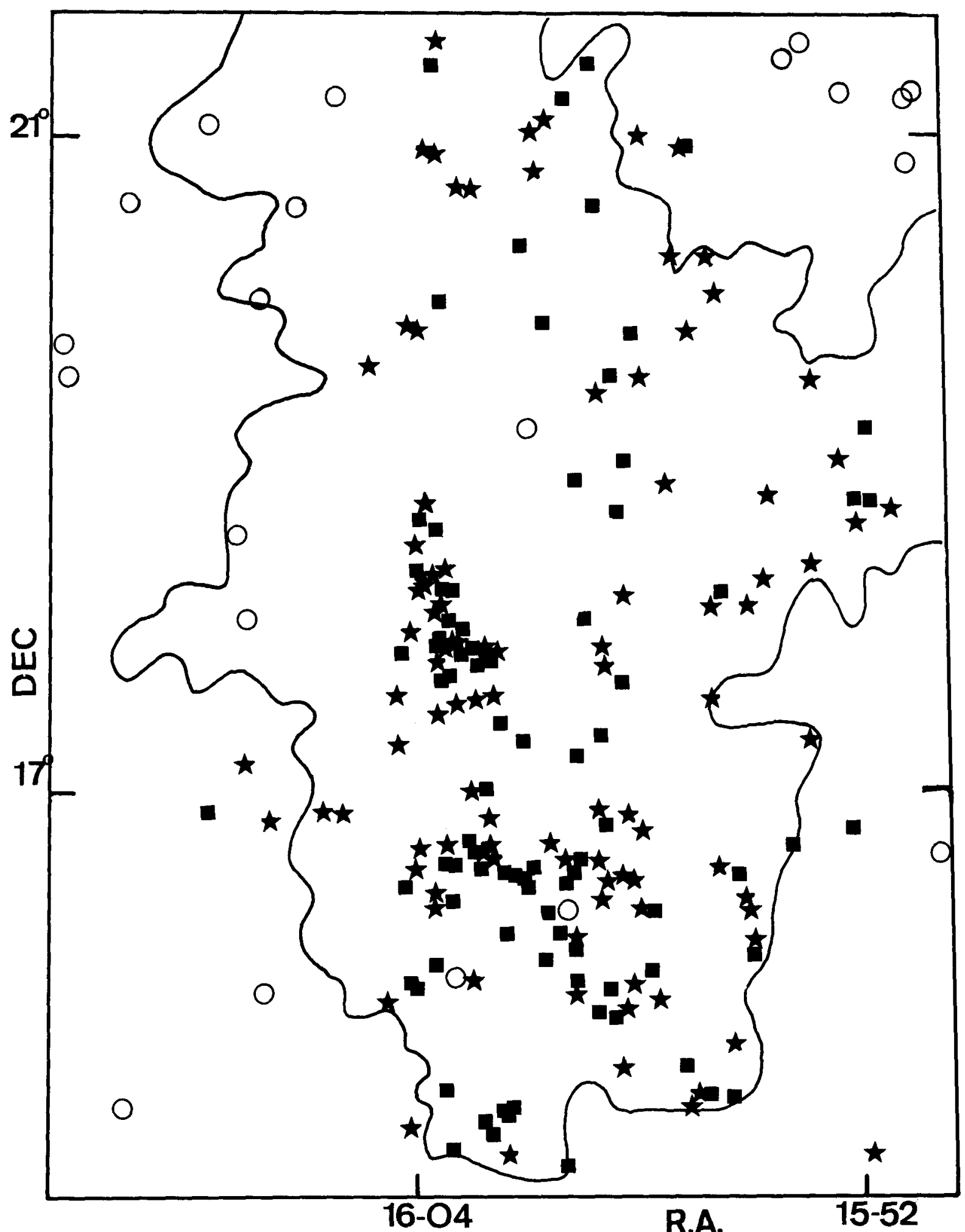

Figure I. Distribution on the sky of the galaxies brighter than $m_{p g}=15.8$. The line countour defines the Zwicky $n^{0} 7$ cluster. Closed squares: $E$ and $S 0$ galaxies. Stars: $S$ and Irr galaxies. open circles: galaxies not classified or of difficult classification. 


\begin{tabular}{|c|c|c|c|c|c|c|c|c|}
\hline & \multicolumn{8}{|c|}{ TABLE I } \\
\hline Name & (1950) & & 950) & $D$ & $\mathrm{R}$ & MAG & $\mathrm{BM}$ & RS \\
\hline 12147 & $16^{\mathrm{h}} 00^{\mathrm{m}} 00^{\mathrm{s}}$ & $16^{\circ}$ & $03^{\circ}$ & 1 & 1 & 13.8 & II & \\
\hline 2151 & $16^{\mathrm{h}} 03^{\mathrm{m}} 00^{\mathrm{s}}$ & $17^{\circ}$ & $53^{\prime}$ & 1 & 2 & 13.8 & III & \\
\hline 12152 & $16^{\mathrm{h}} 03^{\mathrm{m}} 06^{\mathrm{s}}$ & $16^{\circ}$ & $35^{\prime}$ & 1 & 1 & 13.8 & III & \\
\hline
\end{tabular}

We have divided the region under study in approximately two circular subregions: the first one, AREA $I$, centered at $\alpha$ (1950) $=16^{\mathrm{h}} 01^{\mathrm{m}} 30^{\mathrm{s}}$ and $\delta(1950)=17^{\circ} 00^{\prime}$ with a radius of $2^{\circ} .5$, contains the three rich clusters A2147, A2151 and A2152, and the second region AREA II, partly overlapping the former, centered at $\alpha(1950)=15^{\mathrm{h}} 57^{\mathrm{m}} 00^{\mathrm{s}}$ and $\delta(1950)=20053^{\prime} 49^{\prime \prime}$ with a radius of 2.0 degrees, contains the compact group of galaxies known as the Seyfert Sextet. This region has been selected to study the outer parts of the Hercules cluster.

The conventional Hercules cluster, A2151, is an example of irregular cluster. Remarkable aspects are: the high ratio of spirals to elliptical and 50 galaxies, the presence of four conspicuous double systems among the brightest galaxies in the cluster and the peculiar galaxy ICII82, and clampy structure evidencing chains and subgroups. Redshift is known for 18 galaxies. (Burbidge and Burbidge, 1959).

The Abell 2147 cluster is dominated by the peculiar chain Arp 324 (VV159), consisting of two giant $D$ and a number of fainter galaxies, coincident with the apparent cluster center. other chain structures are visible all around, with areas relatively devoid of galaxies in between. A spectroscopic investigation of 16 galaxies in A2147 was done by Bautz (1972). The third rich cluster, A2152, does not present either a center of condensation of galaxies or an outstanding galaxy. There is no clear separation between A2147 and A2152.

\section{MORPHOIOGICAL DATA}

We have a new morphological classification for all the Zwicky's galaxies derived from high quality photographic material. The galaxies in Figure I are shown with separate coding for the E/SO's and the spirals (including the irregulars).

The 202 galaxies in the entire sample are: 17\% E; 27\% s0; $53 \% \mathrm{~S} ; 3 \% \mathrm{Irr} / \mathrm{Pec}$. No large difference is evident in the projected distribution of the E/SO's and of the S/I's. On the other hand a number of differences can be noted if the two rich complexes A2151 and A2147 (+A2152) are compared. In particular: a) A difference in the richness of spirals. L.Thompson (1974) obtained over two areas of 00.7 in radius centered one on A2147 and the other on A2151, considering all galaxies with 
with diameter larger than 15", the following values: A2147= 138 galaxies, $35 \% \mathrm{E}+\mathrm{SO} ; 42 \% \mathrm{~S}+\mathrm{Irr} ; 23 \% \mathrm{SO} / \mathrm{S}$. A2151=159 galaxies, $24 \% \mathrm{E}+\mathrm{SO} ; 65 \% \mathrm{~S}+\operatorname{Irr} ; 12 \% \mathrm{SO} / \mathrm{S}$ where $\mathrm{SO} / \mathrm{s}$ are intermediate galaxies between a so and a spiral. In the same regions considering only the bright galaxies $\left(m_{p g}<15.8\right)$ we have:

A2147:34 galaxies; $56 \% \mathrm{E}+\mathrm{SO}, 41 \% \mathrm{~S}+\operatorname{Irr} ; 3 \%$ SO $/ \mathrm{S}$ A2151:41 galaxies; 46\% E+SO, 54\% S+Irr

b) A difference in the morphology of the brightest members: prevalently spiral in A2151, and ellipticals or SO in A2147 $(+A 2152)$.

c) A difference in the projected space distribution of $\mathrm{E} / \mathrm{SO}$ 's and S/I's. A2151 is centrally concentrated and shows a homogeneous mixture of the two types. A2147 (+A2152) shows no evidence of a central concentration, although elliptical and $S 0$ galaxies seem to be segregated in the inner part. These two clusters appear different in comparison with what observed in other irregular clusters, such as the Virgo cluster (Sandage and Tammann, 1976) and A1367 (Dickens and Moss, 1976) where the distribution of $\mathrm{E}$ and $\mathrm{SO}$ galaxies is noticeably more centrally concentrated.

SPECTROSCOPIC DATA

In order to discriminate between galaxies which are cluster members and field galaxies and to determinate the cluster boundary it is necessary to obtain spectra of a magnitudelimited sample over a large area. At the present time this kind of investigation is available only for two clusters: the Coma Cluster (Tifft and Gregory, 1976; Chincarini and Rood, 1976) and the Virgo Cluster (Sandage and Tammann, 1976). We have therefore started a spectroscopic program over the Hercules cluster region. Within Area $I$, the Zwicky and Herzog catalogue lists 142 galaxies with $\mathrm{m}_{\mathrm{pg}}<15.8$. Sixteen are double and one is triple. Of these, 126 have a magnitude $\mathrm{m}_{\mathrm{pg}}<15.5$. The now available sample can be considered complete to this magnitude since we have velocities for 124 galaxies. 34 were already in the literature, 90 have been observed in the course of this work. To avoid the dangerous of systematic differences among the various sets of observations we also re-observed 15 galaxies with known redshift.

Over both Area I and II we have redshifts for 170 galaxies including 16 not listed by Zwicky and Herzog (1963), and selected because they are Markarian galaxies or faint galaxies identified with radio sources in the Westerbork survey of the Hercules cluster (Jaffe and Perola, 1975). All the new morphological and spectroscopic data will be published in a paper now in preparation by M. Tarenghi, W.G. Tifft, G. Chincarini, H.G. Rood and I. Thompson. Using the redshifts of 119 
galaxies for which the spectroscopic data are already available we obtain the histogram of the velocity distribution shown in Figure 2. Although this is a subsample a few properties can be considered representative because these galaxies are randomly selected.

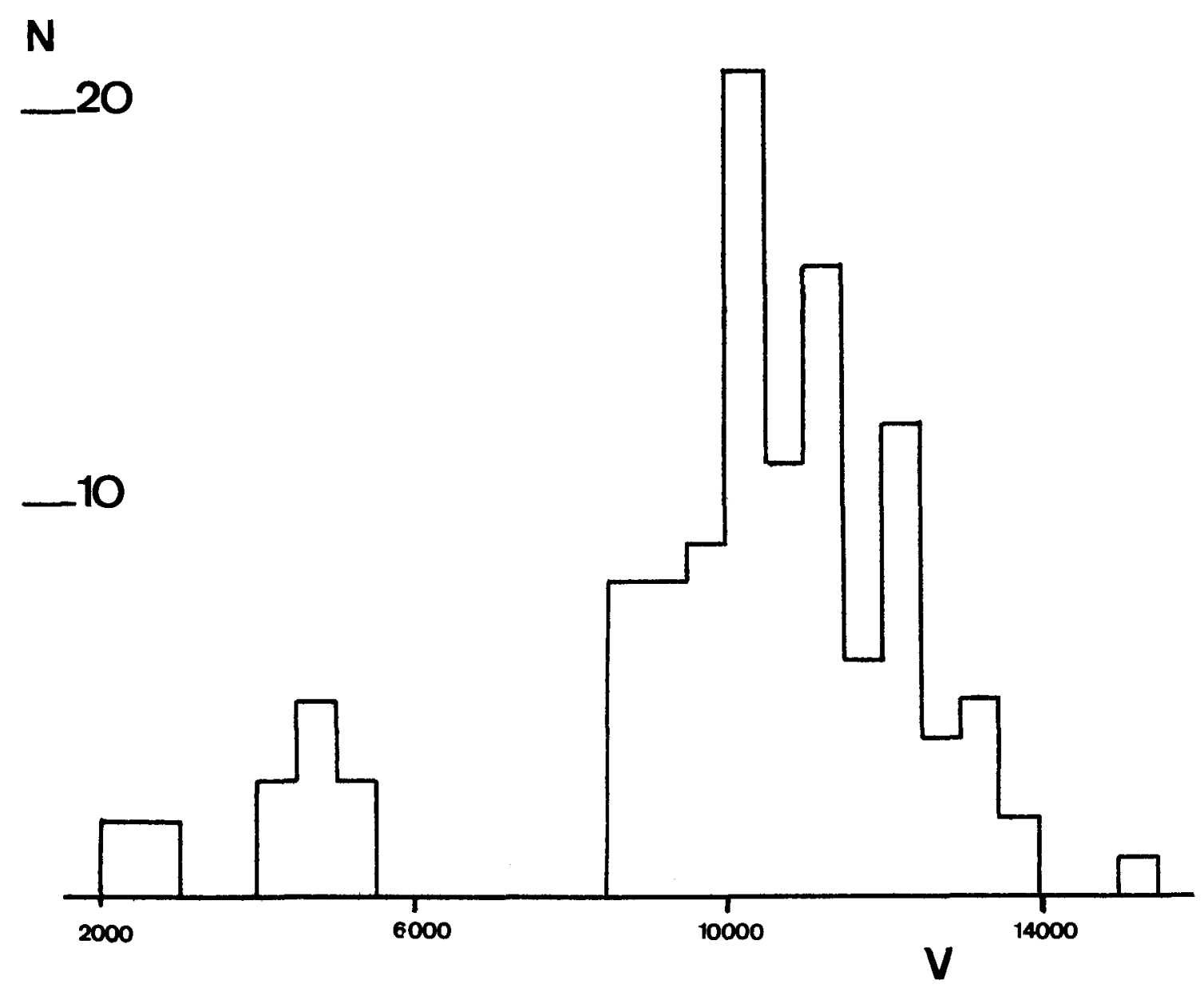

It appears:

Figure 2

1) A well defined segregation of redshifts. In the region of the sky under study the recession velocities of the galaxies down to $\mathrm{m}_{\mathrm{pg}}=15.7$ appear segregated in three main groups corresponding to mean redshifts of: $(a)\langle V\rangle \sim 11000 \mathrm{~km} / \mathrm{sec}$, Hercules cluster complex; (b) $<\mathrm{V}>\sim 4500 \mathrm{Km} / \mathrm{sec}$, a group containing the Seyfert Sextet, and (c) $\langle V\rangle \sim 2300 \mathrm{Km} / \mathrm{sec}$, a nearby group. Note that a segregation of redshifts is also found in the region of the Coma Cluster (Tifft and Gregory, 1976; Chincarini and Rood, 1976), with three groups (7000 $\mathrm{Km} / \mathrm{sec} ; 4500 \mathrm{Km} / \mathrm{sec} ; 1000 \mathrm{Km} / \mathrm{sec})$.

2) There is no evidence of galaxies uniformely distributed in space (the so called field galaxies). 
3) For the galaxies in the range $8500-14000 \mathrm{Km} / \mathrm{sec}$ a mean velocity $\langle\mathrm{V}\rangle \approx 10800 \mathrm{Km} / \mathrm{sec}$, with a dispersion of $\sim 1400$ $\mathrm{Km} / \mathrm{sec}$ is obtained. Note that for A2147 Bautz (1972) found $\langle\mathrm{V}\rangle=11330 \mathrm{Km} / \mathrm{sec}$, with $\sigma=1079 \mathrm{Km} / \mathrm{sec}$, and for A2151 Burbidge and Burbidge (1959) found $\langle V\rangle=10775 \mathrm{Km} / \mathrm{sec}$, with $\sigma=628 \mathrm{Km} / \mathrm{sec}$. The distribution in Figure 2 should be considered a combination of two or more distributions relative to the different subgroups.

The combination of both the morphological and spectroscopic material strongly suggest the existence of a Hercules supercluster. The A2151 and A2147 (+A2152) should be considered as subcondensations of the entire complex.

Abe11,G.0. 1958, Ap. J. Sup. $\underline{3}, 211$

Bautz,I.P. 1972, A.J. 77, 331

Bautz,I.P. and Morgan, W.W. 1970, Ap. J. Letters 162, I149

Burbidge,G.R. and Burbidge,E.M. 1959, Ap. J. 130,629

Chincarini,G. and Rood,H.J. 1976, Ap. J. $206, \frac{130}{30}$

Chincarini,G. and Martins,D. 1975, Ap. J. 196, 335

Jaffe,W.J. and Perola,G.C. 1975, Astron. and Astrophys. Suppl.

21, 137

Rood,H.J. and Sastry,G.I. 1971, Pub. A. S. P. 83, 313

Sandage,A. and Tammann,G.A. 1976, Ap. J. Letters 207, L1

Thompson, I.A. 1974, Ph. D. Dissertation, University of Arizona Tifft,W.G. and Gregory,S.A. 1976, Ap. J. 205, 716

Zwicky,F. and Herzog,E. 1963, Catalogue of Galaxies and Cluster of Galaxies Vol.2 (Pasadena: Cali fornia Institute of Technology) 
DISCUSSION

G. DE VAUCOULEURS: I should like to remind you that I have described this area as the nucleus of the large Hercules Supercluster (cf. Vistas in Astron., 2, 1956). Mr. Corwin has recently estimated the distance.

H. CORWIN: Using de Vaucouleurs' luminosity index $\Lambda$ and new morphological types, luminosity classes, diameters, and photoelectrically measured magnitudes, all reduced to be directly comparable with data in the second Reference Catalogue, a distance modulus of $\mu_{0}=35.1$ ( $\left.\Delta \mathrm{Mpc}=105\right)$ has been derived. This is a preliminary result - in particular, more photometry is needed.

H. ARP: Are you saying that there is a concentration of $2,000 \mathrm{~km} / \mathrm{sec}$, $4,000 \mathrm{~km} / \mathrm{sec}$ and $12,000 \mathrm{~km} / \mathrm{sec}$ galaxies in the Hercules area?

M. TARENGHI: I am saying that over the region studied we have three groups with these mean values.

H. ARP: Then are you saying you do not know whether these various redshift classes are concentrated to the Hercules cluster or not?

M. TARENGHI: The area studied is too small to conclude that the two groups of $2300 \mathrm{~km} / \mathrm{sec}$ and $4500 \mathrm{~km} / \mathrm{sec}$ are or are not concentrated in the direction of the Hercules cluster. There are indications that the $4500 \mathrm{~km} / \mathrm{sec}$ group presents a concentration in the northern part of the region.

J.P. VIGIER: Have you tried to see in your main subsample in redshift whether there is any redshift difference between the average redshifts of spirals and elliptical galaxies?

M. TARENGHI: The present status of the data and the complexity of the region under study do not allow us at the moment to obtain a significant value. It is clear that the results on this point is one of the aims of our research. 